\title{
Protection to thermal impact of solar radiation: evaluation of selected reflective fabrics
}

\author{
Lennart Teunissen ${ }^{1^{*}}$, Linda Plaude ${ }^{1}$, Kaspar Jansen ${ }^{1}$ \\ ${ }^{1}$ Department of Design Engineering, Delft University of Technology, Delft, The Netherlands \\ *Corresponding author E-mail address: lennart.teunissen@gmail.com
}

\section{INFO}

CDAPT, ISSN 2701-939X

Peer reviewed article

2021, Vol. 2, No. 2, pp. 103-114

DOI 10.25367/cdatp.2021.2.p103-114

Received: 03 July 2021

Accepted: 04 September 2021

Available online: 18 September 2021

\section{Keywords}

solar radiation, thermal impact, reflective fabric, heat stress

\begin{abstract}
Prolonged exposure to solar radiation can cause considerable heat stress. The application of reflective materials in garments or sunscreens is generally considered as an appropriate protective strategy. In this study, we aimed to compare a range of reflective and control fabrics on their ability to reduce the thermal impact of solar radiation. We evaluated 16 reflective and 5 control fabrics, varying in applicability for garments and/or sunscreens. Transmission of ultraviolet, visible light and infrared radiation was studied using an artificial solar light. Thermal impact reduction was first studied using artificial infrared light and secondly using natural sunlight, measuring temperature right at the back and $10 \mathrm{~cm}$ behind the fabric after a 10-minute exposure. Most samples showed comparably low radiation transmission (< 10\%). However, substantially higher transmission was observed in perforated and mesh-like reflective fabrics, as well as light colored controls and coldblack $^{\circledR}$ treated fabric. This resulted in larger temperature increases at $10 \mathrm{~cm}$ behind the fabric (+1-4 $\mathrm{K}$ in sunlight). Contact temperature at the back of the black fabrics ended up higher than at the back of the reflective and white control fabrics ( $\triangle T: 5-10 \mathrm{~K}$ in sunlight), the latter two showing minor mutual differences $(\Delta T<$ $3 \mathrm{~K}$ ). In conclusion, the reflective fabrics (excluding perforated, mesh and coldblack $\left.{ }^{\circledR}\right)$ showed minor mutual differences, lower heat absorption than the black control fabrics and lower heat transmission than the white ones. The results suggest that reflective or white fabrics are preferable for most garment applications, while reflective or possibly black fabrics are preferable for sunscreen applications.
\end{abstract}

(C) 2021 The authors. Published by CDAPT.

This is an open access article under the CC BY-NC-ND license https://creativecommons.org/licenses/ peer-review under responsibility of the scientific committee of the CDAPT.

(C) 2021 CDAPT. All rights reserved. 


\section{Introduction}

High ambient temperatures and high levels of humidity create challenging climatic conditions for work and exercise [1-7]. Depending on the clarity of the sky, sunlight radiation may act as an additional source of heat stress. In clear sky with the sun at its zenith, it may deliver a power of $>800 \mathrm{~W} / \mathrm{m}^{2}$ at the earth' surface. These values do not only occur at equatorial areas, but may just as well be attained in highlatitude cities during summer [8]. Heat gain increases with radiation intensity [9], resulting in a higher thermophysiological strain and a progressive reduction in endurance exercise performance $[10,11]$. Therefore protection against radiation by reflective materials may be useful to reduce thermal strain. Probably most effective is providing shade by a reflective sunscreen like a tent or parasol. An alternative is wearing garments with reflective outer layer or reflective elements. There is in fact a wide variety of reflective textile-based materials which claim to be protective. However, it is unclear to what extent these fabrics actually shield from heat transmission. Since there is no a priori way of selecting these fabrics on their heat protection properties, we set up a series of dedicated tests and compared a range of commercially available fabrics.

When evaluating sunlight radiation, it is important to distinguish three different types of radiation, classified according to their wavelength (Table 1). Ultraviolet (UV) radiation that reaches the terrestrial surface consists for a small part of UV-b (290-320 nm) and for the remainder of UV-a (320-400 nm). It is harmful to the skin, being responsible for tanning, burning and skin cancer development [12,13]. UV does not belong to the thermal part of the spectrum, i.e. the part that we experience as heat. Visible light $(V L)$ is the part of the light spectrum we can actually see; depending on the exact reflected wavelengths, we see colors, ranging from blue to red. Absorbed visible light can be converted into heat, but less efficient than infrared. Infrared (IR) radiation is not visible, but experienced as heat on the skin. Therefore, it is also called thermal radiation. All material emits IR radiation, but its intensity depends on the emissivity and the temperature of the material. Sunlight contains mostly IR-A (760-1400 nm) and some IR-B (1400-3000 nm). IR-A penetrates deepest into the skin (up to $5 \mathrm{~mm}$ ), IR-B is less powerful and only heats superficially $[12,14,15]$.

Table 1. Types of sunlight radiation reaching the earth [13].

\begin{tabular}{lll}
\hline Radiation type & Wavelength $(\mathbf{n m})$ & \% of solar spectrum \\
\hline UV & $290-400$ & 7 \\
VL & $400-760$ & 39 \\
IR & $760-3000$ & 54 \\
\hline
\end{tabular}

Radiation can go three ways when falling on an object: transmission ( $T$ ), absorption/emission (E) and reflection $(R)$. Transmission means that radiation penetrates through a material. This characteristic can be different for different radiation types: regarding glass, VL-transmission is high, while IR-transmission is low. Note that many materials are impermeable for IR radiation, its transmission generally only occurs in thin materials [16]. Emissivity is the extent to which a material is able to emit (infrared) radiation. An ideal black globe has a perfect emissivity coefficient of 1 . Non-metals like wood and plastic (but also human skin) have emissivity factors of $0.8-0.98[16,17]$. Reflectivity means that radiation waves bounce back from a material. In materials without transmission, reflectivity is the exact opposite of emissivity $(R$ $=1-\mathrm{E}$ ). So reflective materials have low E-values and do not absorb a lot of radiative energy. Note that during measurements, reflection of external radiation and emission of radiation from the material itself are not always easy to distinguish [16].

For skin and eye protection, it is important that protective fabrics have a low transmission of UV radiation. However, from a thermal perspective, a low transmission and emission of IR radiation is more relevant. After all, low transmission results in less heating of the air or objects behind the material, while low absorption attenuates heating the material itself and its thermal emission to the surroundings. So to attenuate the thermal impact of sunlight radiation, materials should have a low T-value, low E-value and high $\mathrm{R}$-value for IR radiation (and to a lesser extent for $\mathrm{VL}$ ). 
The effects of solar radiation in relation to clothing type and color have been studied quite often [18-24]. However, the thermal effect of using reflective fabrics for protection against solar radiation in comparison to custom fabrics received less frequent attention. Previous research on a thermal manikin reported that reflective fabric attenuates radiative heat gain increasingly across a range of ascending radiation intensities $\left(215-380 \mathrm{~W} / \mathrm{m}^{2}\right)$. At a radiant temperature of $50{ }^{\circ} \mathrm{C}$, heat gain reduction was $75 \%$ for reflective compared to non-reflective materials [9]. Influence of material and color of the non-reflective materials was small. However, this experiment was not focused on short wave IR as emitted by the sun, but on long wave IR as emitted by, e.g., hot surfaces. Other studies suggest that transmission of solar radiant heat to the body could also be reduced by using aluminized clothing [25] or a reflective helmet [26]. Several commercially available reflective fabrics indeed claim to block solar radiation, but it remains unclear to what extent they do reduce transmission and thermal impact. Manufacturer specifications usually lack this specific information, making comparison of products difficult. Therefore, this study investigated a range of reflective and custom fabrics on radiation transmission as well as on attenuation of thermal impact. Thermal impact was determined by measuring the temperature at the back of the material samples and slightly behind, while they were heated by artificial IR light in the lab and natural sunlight in the field. Fabrics were grouped by their suitability for garment or for sunscreen application.

\section{Methods and Materials}

\subsection{Design}

16 reflective and 5 control fabrics, applicable for clothing, caps and/or sunscreens, were evaluated in three experiments. First, radiation transmission was studied using an artificial solar light. Secondly, protection against the thermal impact of IR radiation was studied using an artificial IR light. Thirdly, protection against the thermal impact of solar radiation was studied in natural sunlight. A $30 \mathrm{~cm}$ diameter sample of each fabric was mounted on a cardboard ring for all testing procedures (Fig. 1c-d).

\subsection{Fabrics}

Table 2 lists the investigated reflective and control fabrics usable for garment and/or sunscreen applications. Reflective fabric was broadly defined as fabric being marketed for having reflective properties. For logistic reasons, one fabric was omitted from the second experiment and six from the third experiment, as indicated in the table. Six fabrics were usable for both garment and sunscreen application.

The CSR 2001 and CSR 1303 fabrics were tested in the basic and the perforated version (perforation diameter $\sim 1 \mathrm{~mm}$ ). Coldblack ${ }^{\circledR}$ is not a material itself, but a patented finishing technology for (dark) textiles. When exposed to sunlight over extended periods, the manufacturer claims it reduces heat absorption, preventing dark colors from heating up. In addition it would provide UV protection. The coldblack $®$ finish in this study has been applied on the same black polyester fabric that has been tested as a control. The Kynar Flex sheet was created by dissolving P(VdF-HFP) (Kynar Flex 2801, Arkema) in acetone, adding water to make a $\mathrm{P}(\mathrm{VdF}-\mathrm{HFP})$-acetone-water precursor solution with a 1:8:1 mass ratio. This solution was drop-cast on a substrate to produce a single 'stand-alone' sheet. The material has been reported to provide reflectance and long-wave infrared emittance $(0.97 \pm 0.02)$ from any object it is applied on, making it particularly suited for passive daytime radiative cooling [27].

\subsection{Procedures \& measurements}

\subsubsection{Experiment 1}

Radiation transmission was investigated using a rubber cylinder (30 cm diameter) with two floors. On the cardboard lower floor, a spectrometer (Black-Comet-SR-50, StellarNet Inc, USA) was placed for radiation measurements (range $200-1100 \mathrm{~nm}$, with a resolution of $2 \mathrm{~nm}$ ). 
Table 2. Studied fabrics and coatings for garment and/or sunscreen application.

\begin{tabular}{|c|c|c|c|c|c|c|c|}
\hline Name (reflective) & Composition & Manufacturer / supplier & $\begin{array}{c}\text { Garment } \\
\text { appl }\end{array}$ & $\begin{array}{c}\text { Sunscreen } \\
\text { appl }\end{array}$ & $\begin{array}{c}\text { Exp } \\
1\end{array}$ & $\begin{array}{c}\text { Exp } \\
2\end{array}$ & $\begin{array}{c}\text { Exp } \\
3\end{array}$ \\
\hline Coldblack@ reflective finish & Black polyester + Coldblack@ finish & Schoeller, Switzerland & $\mathrm{x}$ & & $\mathrm{x}$ & $\mathrm{x}$ & $\mathrm{x}$ \\
\hline CSR 2001 fabric & Spandex/Polyester (silver, stretch) & Chinastars Reflective, China & $x$ & & $x$ & $\mathrm{x}$ & $\mathrm{x}$ \\
\hline CSR 2001 fabric perforated & Spandex/Polyester (silver, stretch) & Chinastars Reflective, China & $\mathrm{x}$ & & $\mathrm{x}$ & $\mathrm{x}$ & $\mathrm{x}$ \\
\hline CSR 1303 fabric & Polyester (silver, non-stretch, smooth) & Chinastars Reflective, China & $\mathrm{x}$ & $\mathrm{x}$ & $\mathrm{x}$ & $\mathrm{x}$ & $\mathrm{x}$ \\
\hline CSR 1303 fabric perforated & Polyester (silver, non-stretch, smooth) & Chinastars Reflective, China & $\mathrm{x}$ & $\mathrm{x}$ & $x$ & $x$ & $x$ \\
\hline CSR 3303 fabric & Polyester (silver, non-stretch, rigid) & Chinastars Reflective, China & $\mathrm{x}$ & $\mathrm{x}$ & $\mathrm{x}$ & $\mathrm{x}$ & $\mathrm{x}$ \\
\hline Cool Tech fabric & Aluminum coated glass beads (silver) & Beco Int., South Korea & $x$ & $x$ & $x$ & $x$ & $x$ \\
\hline Flash Tech Silver fabric & Not known (silver) & Sportconfex, $\mathrm{NL}^{*}$ & $x$ & $x$ & $x$ & $x$ & $\mathrm{x}$ \\
\hline Silver roller blind fabric & Not known (silver) & Textile fabric shop, $\mathrm{NL}^{*}$ & $x$ & $x$ & $x$ & $\mathrm{x}$ & $\mathrm{x}$ \\
\hline Duraskin fabric & Duraskin $($ ) comfort plus B1015 (silver) & Verseidag, Germany & & $x$ & $x$ & $x$ & $x$ \\
\hline Tyfek 60 fabric & $\begin{array}{l}\text { Reflex soft structure } 3460 \mathrm{M} \text {, aluminum } \\
\text { coated, } 60 \mathrm{~g} / \mathrm{m}^{2} \text { (silver) }\end{array}$ & Extremtextil, Germany & & $\mathrm{x}$ & $x$ & $x$ & $x$ \\
\hline Alunet fabric single sheet & Mesh reflective material (white) & Création Baumann, NL & & $x$ & $x$ & $x$ & \\
\hline Alunet fabric double sheet & Mesh reflective material (white) & Création Baumann NL & & $x$ & $x$ & $x$ & \\
\hline Eurotrail fabric & Polypropylene (black) & Eurotrail, NL & & $x$ & $x$ & $x$ & \\
\hline Kynar Flex & $\mathrm{P}(\mathrm{VdF}-\mathrm{HFP})$ (Kynar Flex 2801) (white) & Custom made & & $x$ & $x$ & $x$ & \\
\hline \multicolumn{8}{|l|}{ Name (control) } \\
\hline Black polyester fabric & Polyester (black) & Sportconfex, $\mathrm{NL}^{*}$ & $x$ & & $x$ & $x$ & $\mathrm{x}$ \\
\hline Black cotton fabric & Cotton (black) & Textile fabric shop, $\mathrm{NL}^{*}$ & $x$ & & $x$ & $x$ & \\
\hline White cotton fabric & Polyester (white) & Textile fabric shop, $\mathrm{NL}^{*}$ & $x$ & & $x$ & $x$ & \\
\hline
\end{tabular}

${ }^{*}$ Only supplier, manufacturer unknown

The replaceable second floor was formed by one of the sample discs, located at $1 \mathrm{~cm}$ above the radiation sensor. The upper edge of the cylinder extended $5 \mathrm{~cm}$ above this second floor. At $25 \mathrm{~cm}$ above the sample disc, a $80 \mathrm{~W}$ solar light (JBL Spot-Plus, JBL GmbH \& Co, Neufhofen, Germany) was positioned. Four preliminary measurements, collected in different sessions, indicated that the relative contribution of $U V, V L$ and $I R$ in the artificial light was $(6.7 \pm 1.9) \%,(26.9 \pm 7.5) \%$ and $(66.5 \pm 6.1) \%$, respectively.

Fig. 1 provides an illustration of the set-up, showing the first floor with the spectrometer on top of it (1a) and the second floor being one of the sample discs (1b). About a minute after placement of each sample disc, the light spectrum was captured, with absolute $\left(\mathrm{W} / \mathrm{m}^{2}\right)$ and relative $(\%)$ measures of radiative power. The spectrum was categorized into UV-a, UV-b, UV-c and from 400 to $1100 \mathrm{~nm}$ into classes of $100 \mathrm{~nm}$. Prior to the sample discs, a baseline measurement without disc was performed for normalization purposes.

\subsubsection{Experiment 2}

Protection against the thermal impact of IR radiation was investigated using an IR light (InfraCare PR3110/00, $150 \mathrm{~W}$, Philips, The Netherlands) positioned at $50 \mathrm{~cm}$ from a stand, keeping the studied sample disc in a vertical plane. Measurements were performed in a darkened room with a mean ambient temperature of $(26.9 \pm 1.3){ }^{\circ} \mathrm{C}$ across different sessions and negligible air flow.

Temperature at the backside of the sample was measured by a wireless iButton (DS1922L, Maxim Integrated Products Inc, USA), directly in contact with the fabric. iButtons have a mean accuracy of - 
$0.09^{\circ} \mathrm{C}$ with a precision of $0.05^{\circ} \mathrm{C}$ [28]. The iButton was attached to the top of a horizontally placed stick and gently pressed against the fabric, representing a combination of absorbed and transmitted radiative heat. Another iButton on top of a stick was positioned $10 \mathrm{~cm}$ backwards behind the fabric (Fig. 1c), mainly representing transmitted radiative heat alone. Sample rate was $0.1 \mathrm{~Hz}$. Each measurement lasted 10 min, starting by turning on the IR light.

Prior to testing the sample discs, a baseline measurement without disc was performed for reference purposes. In between measurements, iButtons were shortly cooled in water at ambient room temperature to quickly return to a roughly similar baseline temperature.

\subsubsection{Experiment 3}

For a selection of samples, experiment 2 was repeated in a field setting, using natural sunlight as radiation source. Samples were positioned horizontally at $10 \mathrm{~cm}$ above the ground (Fig. 1d). First, selected samples were placed in full sunlight for 10 minutes, while an iButton was attached to the backside of the samples. After cooling down the samples and iButtons to baseline temperature, the same procedure (10 min in full sunlight) was repeated, with the iButtons located $10 \mathrm{~cm}$ below the selected sample discs. Ambient temperature was $26.1{ }^{\circ} \mathrm{C}$ and the set-up was shielded from any natural wind flow (for visibility, shielding was removed on Fig. 1d). Sample rate was $0.1 \mathrm{~Hz}$ again.
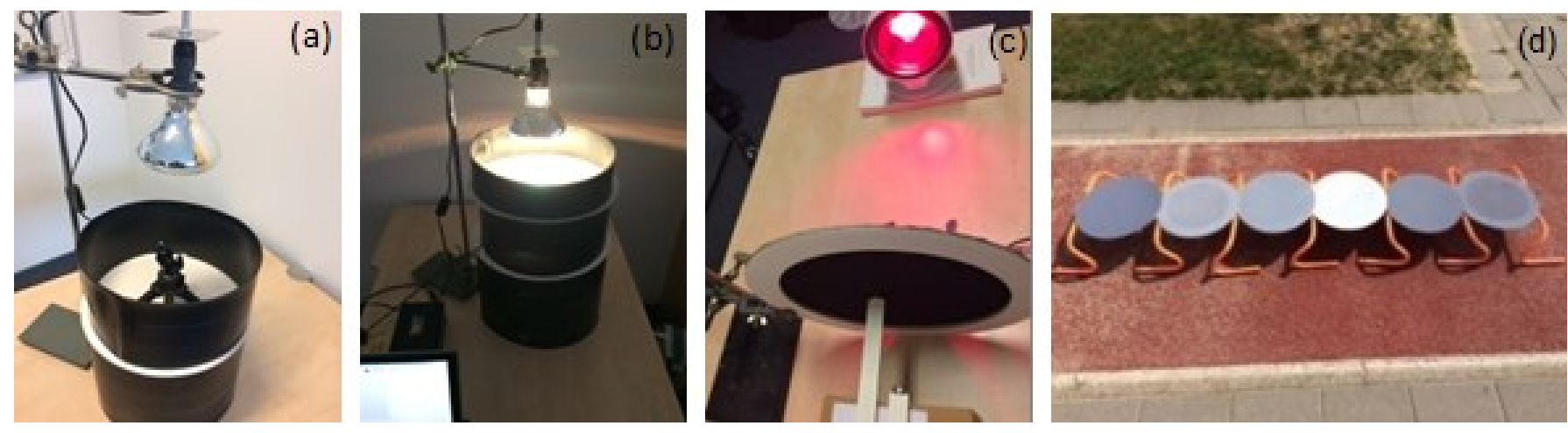

Fig. 1 Set-up of (a) experiment 1 without test sample, (b) experiment 1 with test sample, (c) experiment 2 and (d) experiment 3.

\subsection{Data analysis}

For transmission of UV radiation, the total irradiance $\left(\mathrm{W} / \mathrm{m}^{2}\right)$ of UV-a, UV-b and UV-c was used. For transmission of $V L$ and IR, the total irradiance from 400 to $700 \mathrm{~nm}$ and 700 to $1100 \mathrm{~nm}$, respectively, was determined. Transmission of all three classes of radiation was expressed as percentage irradiance that was transmitted through a sample, compared to the prior baseline measurement without sample coverage.

From experiment 2 and 3, the temperature of both temperature sensors at the end of the intervention phase (average value across the $10^{\text {th }}$ minute after the start of heating) was used for analysis of the garment samples. For the sunscreen samples, only the temperature $10 \mathrm{~cm}$ behind the samples was considered, as the temperature of the fabric itself is less relevant for this application.

Standard deviations across the $10^{\text {th }}$ minute were calculated as a consistency check for all temperature measurements. In addition, polynomial extrapolation of the temperature profile was performed for experiment 2, to check to which extent the measured maximal temperature approached the (theoretical) maximum of the curve. All results are presented for the garment fabrics and sunscreen fabrics separately. Data from fabrics applicable in both categories is presented twice. 


\section{Results and discussion}

\subsection{Transmission}

Fig. 2a shows the relative transmission of $U V, V L$ and $I R$ radiation of the fabrics suited for garment application and Fig. $2 b$ of the fabrics suited for sunscreen application (experiment 1 ). Nearly all samples blocked the bulk of the UV radiation ( $<10 \%$ transmission). An exception concerned the CSR fabrics with perforations and the Alunet mesh fabric, causing part of the radiation to penetrate directly through the fabric. Applying a double sheet of the mesh fabric provided only a partial reduction. Further, the white control fabrics were somewhat less protective than the black and reflective ones, in line with more extensive research on this topic [18, 24].

The transmission of VL shows a similar picture, although the higher transmission of the white control fabrics is much stronger on these wavelengths. The white cotton and polyester control fabrics transmit a substantial amount of VL (37 and 69\%, respectively), while the white Kynar Flex coating still transmits a moderate amount (17\%). This is due to the lack of VL absorption, in contrast to colored fabrics.
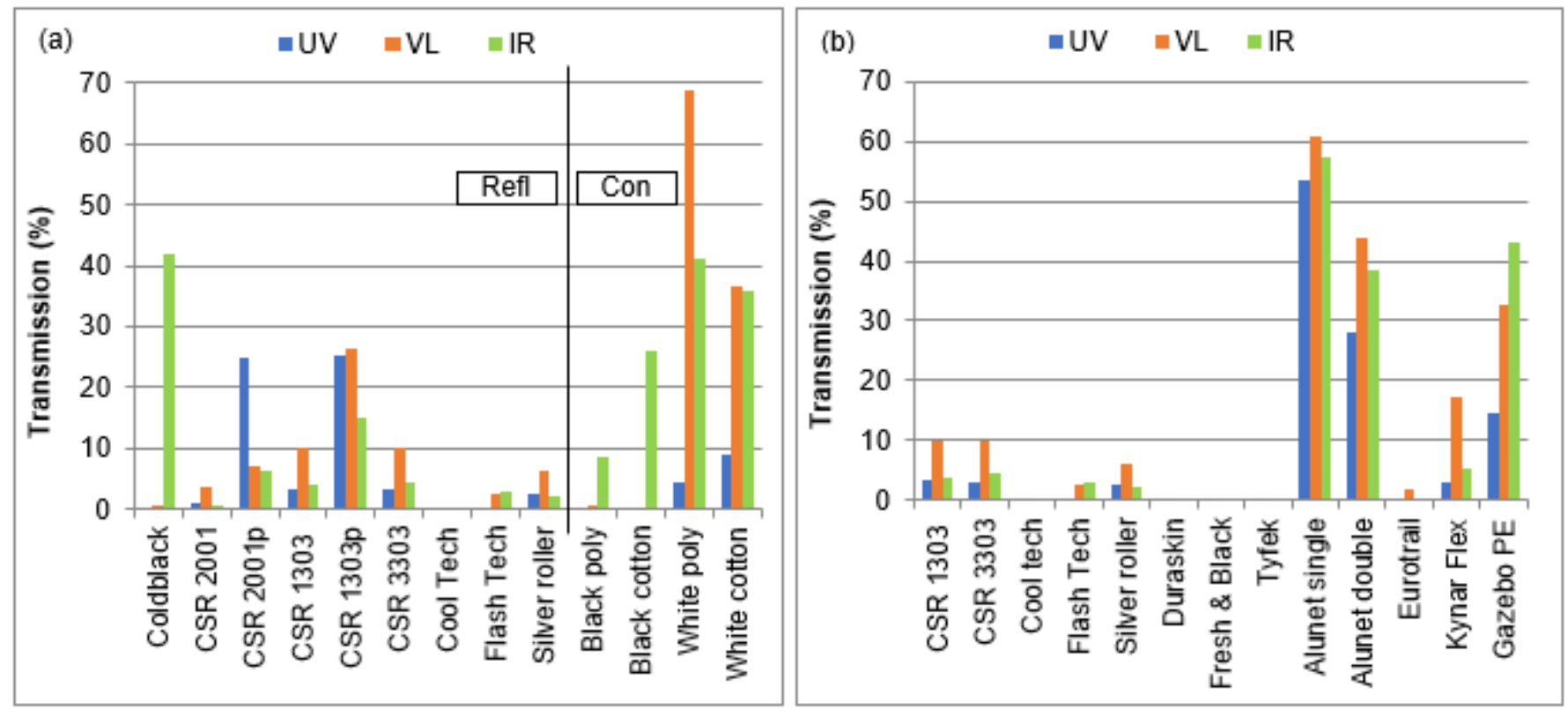

Fig. 2 Relative transmission of UV, VL and IR radiation for (a) the garment fabrics and (b) the sunscreen fabrics. The left 9 garment fabrics are reflective, the four fabrics on the right are non-reflective controls. For the sunscreen fabrics, only Gazebo PE is a non-reflective control fabric. Invisible columns indicate a (nearly) $0 \%$ transmission.

Transmission of IR is particularly high for the white control fabrics (36-41\%) as well as the Alunet mesh fabric and PE control (38-57\%). The black control fabrics transmit IR to a somewhat lower extent $(<30 \%)$. However, the coldblack ${ }^{\circledR}$ finish increases transmission of IR radiation considerably (from $9 \%$ without to $42 \%$ with finish), apparently channeling the originally absorbed IR radiation through the fabric. All other reflective fabrics without perforations transmitted very little IR radiation $(<5 \%)$. Overall, the two best performing reflective garment fabrics in this experiment are CoolTech and CSR2001. For the sunscreen fabrics the top four consists of CoolTech, Duraskin, Fresh\&Black and Tyfek.

\subsection{Thermal impact}

Fig. 3a shows the contact temperature at the backside of the garment fabric samples after $10 \mathrm{~min}$ exposure to an IR light in the lab (experiment 2) and to natural sunlight in the field (experiment 3). Cool Tech and CSR2001 fabric showed the lowest temperatures, apparently having the most favorable 
combination of low transmission and low absorption. In natural sunlight, the highest values were recorded at the black fabrics, which absorb most radiation $(5-10 \mathrm{~K}$ higher contact temperature for coldblack $\AA$ and black poly). The reflective fabrics and white polyester showed minor mutual differences $(<3 \mathrm{~K})$, but did not all have a positive effect compared to the baseline measurement without (contact to) a sample disc. As transmission is low for all of these fabrics, this effect is probably due to different heat absorption and conduction properties of the materials.

The fact that the contact temperature at the back of the coldblack $\circledast$ and white cotton samples only slightly increased after IR light exposure, despite their high transmission of IR, suggests that this measurement is predominantly determined by heat absorption of the fabric and to a minor extent by transmission. Further, it is notable that the test with IR light resulted in a different temperature spectrum across fabrics than the sunlight test. The additional VL radiation in sunlight had a fabric dependent thermal effect, which impacted the backside of the black fabrics, most presumably due to their VL absorption. It stresses the importance of performing specific testing in the environmental conditions of interest.

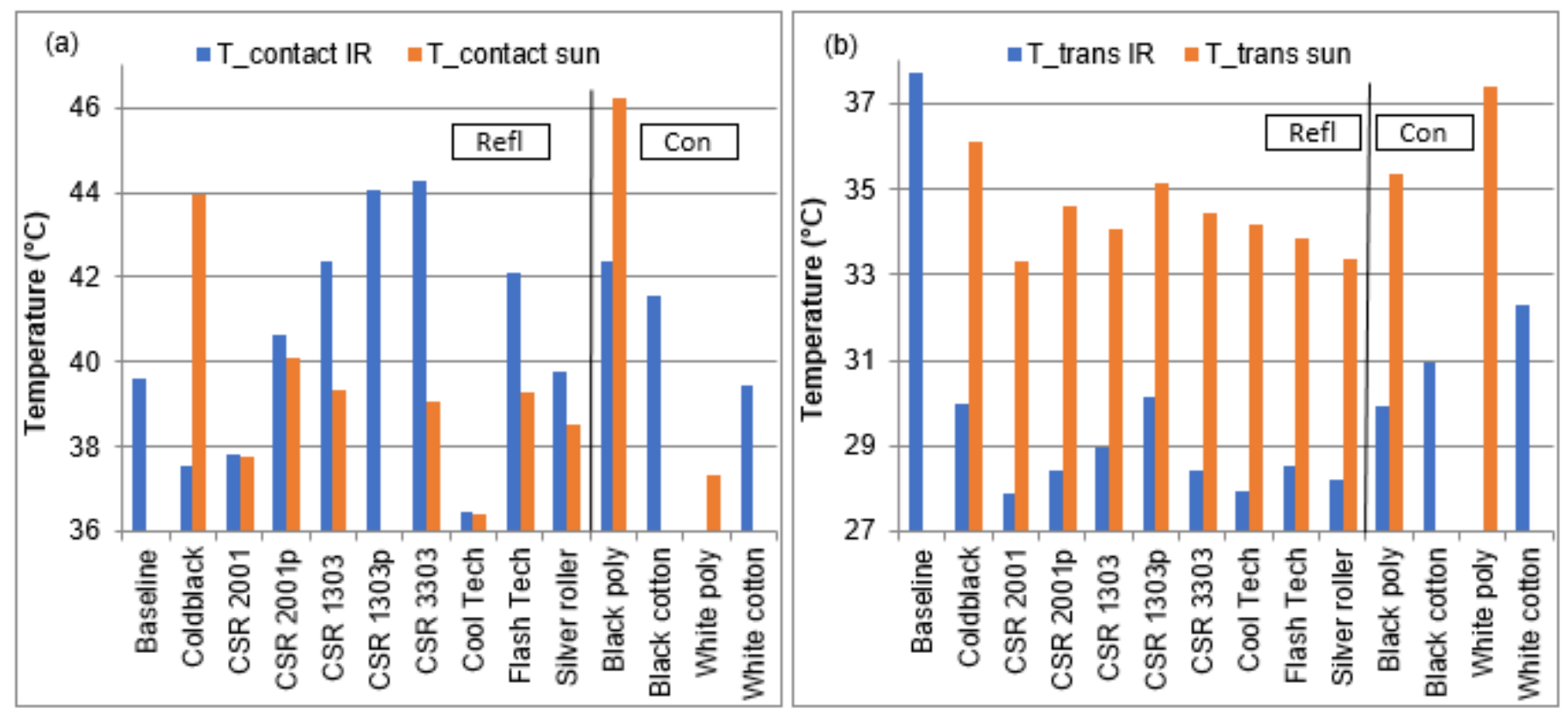

Fig. 3 (a) Contact temperature at the backside of the garment fabrics and (b) temperature $10 \mathrm{~cm}$ behind the garment fabrics, both after 10 min exposure to an IR light in the lab (IR) and to natural sunlight in the field (sun). The four labels most right are control fabrics. Missing columns indicate missing data.

Fig. $3 \mathrm{~b}$ shows the temperature $10 \mathrm{~cm}$ behind the garment fabric samples, roughly reflecting the extent of transmitted IR radiation. The baseline measurement in IR light (100\% IR transmission) indicated that the reflective fabrics mitigated the temperature increase $10 \mathrm{~cm}$ behind about $8-10 \mathrm{~K}$, while the control fabrics still prevented 5-7 K temperature increase. CSR2001 and silver roller fabric remained coolest. The higher IR transmission resulted in a 1-4 $\mathrm{K}$ larger temperature increase in natural sunlight for the perforated reflective fabrics (compared to unperforated), white polyester and coldblack $®$ treated fabric. Again, the relative differences between the IR test and the sunlight test are largely explicable by the thermal effect of transmitted VL.

Fig. 4 is similar to Fig. 3b, but for the fabrics with sunscreen application. In accordance with the garment fabrics, most reflective fabrics strongly mitigate temperature increase $10 \mathrm{~cm}$ behind $(>8 \mathrm{~K}$ in IR light, except for Alunet). This is considerably more than the gazebo PE non-reflective control fabric, mitigating temperature increase by only $\sim 2 \mathrm{~K}$ because of its high IR transmission. Tyfek provided the best heat protection in both IR and natural sunlight, although differences among most reflective fabrics were modest. 


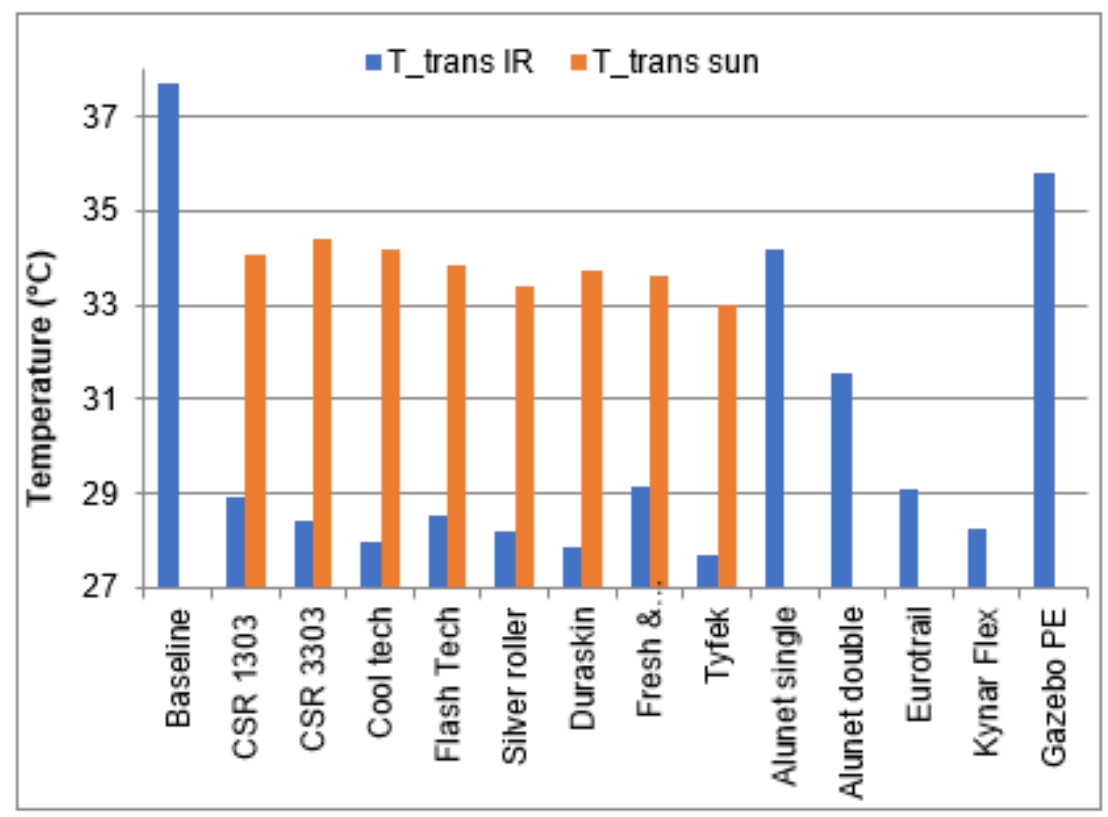

Fig. 4 Temperature $10 \mathrm{~cm}$ behind the sunscreen fabrics after 10 min exposure to an IR-light in the lab (T_trans IR) and to natural sunlight in the field (T_trans sun). Only Gazebo PE is a non-reflective control fabric. Missing columns indicate missing data.

With regard to measurement variability, the average SD within the last $\left(10^{\text {th }}\right)$ minute of temperature recording was $<0.12 \mathrm{~K}$ for the T_contact measurements and $<0.06 \mathrm{~K}$ for the T_trans measurements, indicating an appropriate consistency. Further, the maximum of the polynomial fit deviated only $(0.2 \pm 0.2) \mathrm{K}$ and $(0.5 \pm 0.5) \mathrm{K}$ from the measured maximum for T_contact and T_trans, respectively. We consider this a confirmation that temperature had stabilized to an acceptable level.

In general, the reflective fabrics (excluding perforated, mesh and coldblack $®$ ) showed only minor differences between each other in natural sunlight ( $\Delta \mathrm{T}$ at fabric $<3 \mathrm{~K} ; \Delta \mathrm{T} 10 \mathrm{~cm}$ behind $<1.8 \mathrm{~K}$ ), had a lower heat absorption than the black control fabrics and lower heat transmission than the white control fabrics. This is in accordance with previous research, reporting that wearing a reflective fabric [9] or helmet [26] leads to a lower radiative heat gain of the body compared to their non-reflective counterparts. So using reflective materials, limiting both absorption and transmission, is considered most optimal.

Next to reflective fabrics, white fabrics maintained a far lower contact temperature in sunlight than black fabrics due to their low radiative absorption. Nevertheless, the relatively high radiative transmission did lead to a slightly higher temperature at $10 \mathrm{~cm}$ behind the white than behind the black control fabrics (Fig. 3 ). So temperature data of elements in contact with the fabric (or directly underneath) are rather opposite to those measured at some distance. This is in accordance with previous analyses: on the one hand, the smaller absorbance of radiation in light colored clothing causes a smaller temperature increase in and under neutral fit clothing [20,22] and hats [23], but on the other hand, black clothing can provide better thermal comfort when there is sufficient space between the outer and inner cloth (or skin) [19]. Actually, the latter is also related to increased free convection underneath warmer fabric and explains the use of dark clothing in some equatorial desert regions [19].

Black polyester with a coldblack $\circledast$ treatment (claiming to prevent black garment to become hot) indeed kept the fabric cooler than without coldblack ${ }^{\circledR}$ treatment. However, transmission of thermal radiation (IR) through the fabric increased simultaneously, raising the temperature $10 \mathrm{~cm}$ behind. So although it shifted the radiative behavior of the black fabric a little more to the white side, both the positive and negative mechanisms will determine the net physiological or perceptual benefit. A similar picture was observed at the reflective materials with an open (perforated or mesh-like) structure: higher transmission of radiation, leading to a higher heat transfer to objects/surfaces at some distance behind the fabric. 


\subsection{Practical implications and recommendations}

Solar radiation is known to affect human heat strain; progressive increases in skin temperature, sweat rate and thermal perception have been reported $[10,11,29,30]$. Consequently, greater cardiovascular strain, fatigue and performance loss will be observed [31,32]. Prolonged exposures during work or sports will be affected most. Downs [33] calculated that during the Tokyo Olympics, a gold medalist in tennis, golf and road cycling will have the highest radiation exposure. In these types of activity, proper protection is warranted. Some practical implications can be drawn from the current results:

\subsubsection{Fabric selection}

As stated in the introduction, it is often difficult to objectively compare and select reflective fabrics on their protection against thermal impact. Based on the current measurement, the Cooltech fabric was seen to perform best in the category of the garment fabrics. It had virtually no UV, VL and IR transmission and a minimum temperature increase as measured at the fabric backside. CSR2001 and FlashTech can be considered as second best, while the perforated and treated fabrics were less favorable. Comparing the sunscreen fabrics, Tyfek could be appointed as best choice, although differences were small. The lack of substantial differences between the individual reflective materials suggests that other considerations than thermal impact could be decisive in the definite choice.

Regarding fabric color, the current results indicate that white fabrics are still to be preferred over black fabrics for garment applications with a tight to neutral fit. For sunscreen applications like tents or umbrellas, black colored fabrics are likely to outweigh the white ones due to lower heat transmission. The reflective materials combine the white benefit of low heat absorption and the black benefit of low heat transmission. However, they did not clearly outperform the white controls on contact temperature (Fig. 3a) nor the black controls on temperature $10 \mathrm{~cm}$ behind the fabric (Fig. 3b). Therefore further research should reveal whether their use provides a meaningful physiological benefit over white fabrics for garments or over black fabrics for sunscreens at all. It should also be addressed to what extent these results can be generalized to other light and dark colors. In addition, other transmission determining factors like fiber type, fabric mass and use of dye [18,21,24] could be taken into account. Regarding sunscreens, it would also be relevant to look at the decrease of thermal impact with distance; in practice subjects will often be further from the fabric than the currently studied $10 \mathrm{~cm}$.

\subsubsection{Convection vs. radiation protection}

To improve convective and evaporative heat loss, (reflective) garments may be provided with additional air channels. The results of the mesh-type Alunet fabrics or the perforated CSR2001p and CSR1303p fabrics indicate that this improvement of breathability increases the exposure to radiant heat stress. It is therefore recommended to look for an optimal trade-off between the conflicting effects of radiation protection and convective heat loss or to look for smart design solutions. A relevant example of the latter can be found in headgear. The head is highly exposed to solar radiation but also requires air flow at the same time. Previous research on bicycle helmets showed that $50-75 \%$ of radiant heat gain can be shielded. However, the size of the air vents exposing the head to radiation, is positively related to the radiant heat gain [34]. A helmet design using a laminar system, creating an optically closed surface relative to the sun, managed to prevent radiant heat gain by $95 \%$ while limiting the convective heat loss capacity of the bare head by only $9 \%$ [26]. Another option is choosing for a cooling cap using conductive cooling by an inner lining of phase change materials. In such a case, air vents are not required and a full reflective outer lining can be applied to prevent radiant heat gain of the PCM, considerably enlarging depletion time.

A similar trade-off may arise in the choice between short and long arm sleeves. In warm conditions with intense radiation, there will be a net radiant heat gain of the body in relation to the environment. Current results indicate that long white or reflective sleeves can protect well against radiant heat gain. Although it 
partly blocks convective/evaporative cooling, this radiant protection may be prioritized in certain conditions. This may for example be the case in warm and humid conditions, when convective/evaporative cooling is low anyway, or during short high-intensity exercise, when a positive thermal perception and optimal aerodynamics (due to long sleeves) are more relevant than cooling.

\subsection{Limitations of the study}

First, this study was limited to material testing and did not involve the complex thermoregulatory responses of the human body in interaction with garment or a sunscreen. Therefore, absolute temperature data will differ from real-life human skin values. However, the found material properties may provide a first guidance in selecting radiation protective fabrics and should be validated by physiological and perceptual human subject testing. Secondly, the power spectral profile of the IR light will have been somewhat deviant from the IR radiation in solar light. Data of experiment 2 and 3 should therefore not be compared in an absolute sense, but only relative to each other across fabrics. In future studies, it is recommended to measure as close as possible to real-life conditions, including baseline and repeated measurements for increased significance. Thirdly, it cannot be excluded that free convection and radiation have had some influence on the iButtons. However, as air flow was minimized, ambient temperature was relatively high and conditions were equal for all fabrics, its effect on the main results is considered limited.

At last, some remarks need to be made on the contact temperature measurements in experiment 2 and 3. Due to the low thermal capacity and low heat conduction in the fabric plane, it is difficult to measure the absolute temperature of the fabric surface accurately. In addition, the iButton in contact with the fabric will not only be heated by the fabric's absorbed radiation but also by the transmitted radiation. This measurement therefore only aims to provide an indication of the contact temperature change at the back of the fabric, not an exact representation of the fabric's temperature. Nevertheless, Fig. 3 suggests that this parameter does predominantly track the amount of absorbed radiation (in view of the fabric dependent influence of $V L$ ) and less the transmitted radiation (in view of the limited agreement with IR transmission in Fig. 2). In future measurements, thin heat flow sensors might be attached to the thermostatic surface for increasing accuracy and repeatability of these measurements.

\section{Conclusion}

The investigated reflective fabrics showed low transmission values on all wavelengths with only minor mutual differences, except for the perforated, mesh and coldblack® treated fabrics. In general, temperature data demonstrated that the reflective fabrics had a lower heat absorption than the black fabrics (5-10 K lower contact temperature in natural sunlight) and lower heat transmission than the white control fabrics ( $\sim 3 \mathrm{~K}$ lower temperature $10 \mathrm{~cm}$ behind the sample). This suggests that reflective or light fabrics are preferable over black fabrics for most garment applications; reflective or possibly black fabrics are preferable for sunscreen applications. Cooltech performed best in the garment category showing minimal transmission and the lowest temperature increase at the fabric backside. Tyfek performed best in the sunscreen category showing no transmission and the lowest temperature increase $10 \mathrm{~cm}$ behind the fabric.

\section{Funding}

This work was supported by The Netherlands Organisation for Health Research and Development (ZonMW) [grant number 546001003] and The Dutch Research Council (NWO) [grant number P16-28].

\section{Declaration of conflicting interests}

The authors declare that there is no conflict of interest. 


\section{References}

[1] S. D. R. Galloway and R. J. Maughan. 1997. Effects of ambient temperature on the capacity to perform prolonged cycle exercise in man. Medicine and Science in Sports and Exercise 29, 1240-1249.

[2] J. Gonzalez-Alonso, C. Teller, S. L. Andersen, F. B. Jensen, T. Hyldig, and B. Nielsen. 1999. Influence of body temperature on the development of fatigue during prolonged exercise in the heat. J. Appl. Physiol. 86, 3, 10321039.

[3] R. J. Maughan, H. Otani, and P. Watson. 2012. Influence of relative humidity on prolonged exercise capacity in a warm environment. European Journal of Applied Physiology 112, 6, 2313-2321.

[4] S. J. Petruzzello, J. I. Gapin, E. Snook, and D. L. Smith. 2009. Perceptual and physiological heat strain: examination in firefighters in laboratory- and field-based studies. Ergonomics 52, 6, 747-754.

[5] A. D. Flouris, P. C. Dinas, L. G. Ioannou, L. Nybo, G. Havenith, G. P. Kenny, and T. Kjellstrom. 2018. Workers' health and productivity under occupational heat strain: a systematic review and meta-analysis. Lancet Planet Health 2, 12, e521-e531.

[6] R. A. Lucas, Y. Epstein, and T. Kjellstrom. 2014. Excessive occupational heat exposure: a significant ergonomic challenge and health risk for current and future workers. Extrem. Physiol. Med. 3, 14.

[7] B. Nielsen, T. Hyldig, F. Bidstrup, J. Gonzalez-Alonso, and G. R. Christoffersen. 2001. Brain activity and fatigue during prolonged exercise in the heat. Pflugers Arch. 442, 1, 41-48.

[8] F. Lindberg, B. Holmer, S. Thorsson, and D. Rayner. 2014. Characteristics of the mean radiant temperature in high latitude cities - implications for sensitive climate planning applications. Int. J. Biometeorol. 58, 5, 613-627.

[9] P. Brode, K. Kuklane, V. Candas, E. A. Den Hartog, B. Griefahn, I. Holmer, H. Meinander, W. Nocker, M. Richards, and G. Havenith. 2010. Heat gain from thermal radiation through protective clothing with different insulation, reflectivity and vapour permeability. International Journal of Occupational Safety and Ergonomics: JOSE 16, 2, 231-244.

[10] H. Otani, M. Kaya, A. Tamaki, H. Goto, K. Tokizawa, and R. J. Maughan. 2020. Combined effects of solar radiation and airflow on endurance exercise capacity in the heat. Physiol. Behav. 113264.

[11] H. Otani, M. Kaya, A. Tamaki, P. Watson, and R. J. Maughan. 2016. Effects of solar radiation on endurance exercise capacity in a hot environment. European Journal of Applied Physiology 116, 4, 769-779.

[12] A. M. Holzer, M. Athar, and C. A. Elmets. 2010. The other end of the rainbow: infrared and skin. Journal of Investigative Dermatology 130, 6, 1496-1499.

[13] I. E. Kochevar, M. A. Pathak, and J. A. Parrish. 1999. Photophysics, photochemistry, and photobiology. In: I.M. Freedberg, A.Z. Eisen, K. Wolff, K.F. Austen, L.A. Goldsmith, S.I. Katz, T.B. Fitzpatrick (Eds.) Fitzpatrick's Dermatology in General Medicine (5th. ed.). McGraw-Hill, New York, NY, 220-229.

[14] Bundesamt für Strahlenschutz. Applications of infrared radiation. 2020. Retrieved July 2021 from https://www.bfs.de/EN/topics/opt/application-medicine-wellness/infrared/infrared_node.html.

[15] S. M. Schieke, P. Schroeder, and J. Krutmann. 2003. Cutaneous effects of infrared radiation: from clinical observations to molecular response mechanisms. Photodermatology, Photoimmunology \& Photomedicine 19, 5, 228-234.

[16] P. H. Cornelissen. Warmtebeelden interpreteren. 2011, 1-20. Retrieved Aug 2020 from http://img.warmtecheck.nl/Warmtebeelden\%20interpreteren.pdf.

[17] J. Steketee. 1973. Spectral emissivity of skin and pericardium. Physics in Medicine and Biology 18, 5, 686694.

[18] S. Davis, L. Capjack, N. Kerr, and R. Fedosejevs. 1997. Clothing as protection from ultraviolet radiation: which fabric is most effective? International Journal of Dermatology 36, 5, 374-379.

[19] L. Hes, K. Bal, and M. Boguslawska-Baczek. 2014. Why black clothes can provide better thermal comfort in hot climate than white clothes. In Proceedings of the The Fiber Society Spring Conference, May 21-23, 2014, Liberec, Czech Republic. DOI:10.13140/2.1.1981.5049.

[20] W. Ruchtie. 2018. Effect of cycling shirt properties on thermal strain. Master's thesis. VU University, Amsterdam, The Netherlands.

[21] S. Kuwabara, K. Joko, and K. Takahashi. 2016. Promotion of heat retaining of clothes by dyeing cloth with a dye absorbing infrared rays. Journal of Textile Engineering 62, 3, 43-50.

[22] Y. Shimazaki, S. Goto, A. Yoshida, and T. Yamamoto. 2017. The effect of solar radiation on temperature distribution in outdoor human-clothing-environment systems. International Journal of Heat and Mass Transfer 104, 1-6.

[23] J. Smith and W. Throop. 2006. The effect of color on temperatures inside hardhats. Tech Tip 0651-2312MTDC. Department of Agriculture, Forest Service, Missoula Technology and Development Center, Missoula, MT.

[24] C. A. Wilson, N. K. Bevin, and R. M. Laing. 2008. Solar protection - effect of selected fabric and use characteristics on ultraviolet transmission. Textile Research Journal 78, 2, 95-104. 
[25] C. P. Bogerd, J.-M. Aerts, S. Annaheim, P. Brode, G. de Bruyne, A. D. Flouris, K. Kuklane, T. Sotto Mayor, and R. M. Rossi. 2015. A review on ergonomics of headgear: Thermal effects. International Journal of Industrial Ergonomics 45, 1-12.

[26] Y. Shapiro, D. Moran, Y. Epstein, L. Stroschein, and K. B. Pandolf. 1995. Validation and adjustment of the mathematical prediction model for human sweat rate responses to outdoor environmental conditions. Ergonomics 38, 5, 981-986.

[27] C. P. Bogerd, P. A. Bruhwiler, and R. Heus. 2008. The effect of rowing headgear on forced convective heat loss and radiant heat gain on a thermal manikin headform. J. Sports Sci. 26, 7, 733-741.

[28] J. Mandal, Y. Fu, A. C. Overvig, M. Jia, K. Sun, N. N. Shi, H. Zhou, X. Xiao, N. Yu, and Y. Yang. 2018. Hierarchically porous polymer coatings for highly efficient passive daytime radiative cooling. Science 362, 6412, 315-319.

[29] W. D. Van Marken Lichtenbelt, H. A. Daanen, L. Wouters, R. Fronczek, R. J. Raymann, N. M. Severens, and E. J. Van Someren. 2006. Evaluation of wireless determination of skin temperature using iButtons. Physiol. Behav. 88, 4-5, 489-497.

[30] S. G. Hodder, and K. Parsons. 2007. The effects of solar radiation on thermal comfort. Int. J. Biometeorol. 51, 3, 233-250.

[31] B. Nielsen, K. Kassow, and F. E. Aschengreen. 1988. Heat balance during exercise in the sun. European Journal of Applied Physiology and Occupational Physiology 58, 1-2, 189-196.

[32] S. N. Cheuvront, R. W. Kenefick, S. J. Montain, and M. N. Sawka. 2010. Mechanisms of aerobic performance impairment with heat stress and dehydration. J. Appl. Physiol. 109, 6, 1989-1995.

[33] M. N. Sawka, S. N. Cheuvront, and R. W. Kenefick. 2012. High skin temperature and hypohydration impair aerobic performance. Experimental Physiology 97, 3, 327-332.

[34] N. J. Downs, T. Axelsen, P. Schouten, D. P. Igoe, A. V. Parisi, and J. Vanos. 2020. Biologically effective solar ultraviolet exposures and the potential skin cancer risk for individual gold medalists of the 2020 Tokyo Summer Olympic Games. Temperature (Austin) 7, 1, 89-108.

[35] P. A. Bruhwiler. 2008. Radiant heat transfer of bicycle helmets and visors. J. Sports Sci. 26, 10, 1025-1031. 